\title{
Taming Molecular Beams
}

Sebastiaan Y.T. van de Meerakker, Hendrick L. Bethlem* and Gerard Meijer**

Fritz-Haber-Institut der Max-Planck-Gesellschaft, Faradayweg 4-6, D-14195

Berlin, Germany

* Laser Centre Vrije Universiteit, De Boelelaan 1081, NL-1081 HV

Amsterdam, the Netherlands

**e-mail: meijer@fhi-berlin.mpg.de

The motion of neutral molecules in a beam can be manipulated with inhomogeneous electric and magnetic fields. Static fields can be used to deflect or focus molecules, while time-varying fields can be used to decelerate or accelerate beams of molecules to any desired velocity. We review the possibilities that this molecular beam technology offers, ranging from ultra-high resolution spectroscopy using molecular fountains to novel crossed beam scattering experiments.

\section{Introduction}

Atomic and molecular beams have played central roles in many experiments in physics and chemistry - from seminal tests of fundamental aspects of quantum mechanics to molecular reaction dynamics - and have found a wide range of applications [1]. Nowadays, sophisticated laser-based detection methods exist to selectively detect molecules in specific quantum states. In the early days, quantum state selectivity in the detection process was achieved by inhomogeneous magnetic and/or electric field sections to influence the trajectories of the particles to the detector. This was the approach used by Otto Stern and Walther Gerlach in 1922 [2], and the key concept of their experiment, i.e. the sorting of quantum states via space quantization, has been extensively used ever since. The original experimental geometries were devised to create strong magnetic or electric field gradients on the beam axis to efficiently deflect particles [3]. Later, both magnetic [4, 5] and electric [6] field geometries were designed to focus particles in selected quantum states onto the detector. An electrostatic quadrupole focuser was used to couple a beam of ammonia molecules into a microwave cavity. The resulting inverted population distribution led to the invention of the maser by Gordon, Zeiger and Townes in 1954-1955 [7, 8]. By using several multipole focusers in succession, with interaction regions with electro-magnetic radiation in between, versatile set-ups to unravel the quantum structure of atoms and molecules were developed. In scattering experiments, multipole focusers in combination with electrostatic orientation fields were exploited to study steric effects, i.e., "to aim the molecular arrow" [9]. Variants of these methods were implemented in many laboratories, and have yielded a wealth of detailed information on stable molecules, radicals and molecular complexes, thereby contributing enormously to our present understanding of intra- 
and inter-molecular forces.

The manipulation of beams of atoms and molecules with electric and magnetic fields is thus about as old as the field of atomic and molecular beams itself, and it actually has been crucial for the success of the latter field. However, this manipulation exclusively involved the transverse motion of the molecules ${ }^{1}$. It was only in 1999, that it was experimentally demonstrated that appropriately designed arrays of electric fields in a so-called 'Stark decelerator' can also be used to influence and control the longitudinal (forward) velocity of the molecules in a beam, e.g. to decelerate a beam of neutral polar molecules [14]. Since then a variety of decelerators have been designed and built, ranging in size from decelerators integrated on a chip to several meter long decelerators, and have made a whole variety of new experiments possible. For instance, when the molecules are decelerated to a standstill, they can be loaded and confined in traps. This allows the observation of molecules in complete isolation from their environment for times up to several seconds, and enables the investigation of molecular properties and their interactions in unprecedented detail. The subject of this Review is how full control over the 3D motion of neutral molecules can be achieved, and how these tamed molecular beams can be used to advantage in a variety of novel experiments.

\section{The Stark decelerator and trap}

The Stark decelerator for neutral polar molecules is the equivalent of a linear accelerator for charged particles. Whereas in charged particle accelerators the force that is exerted on a particle depends on its charge and the electric field strength, in a Stark decelerator, the dipole (charge times separation) in a polar molecule is acted upon by electric field gradients. This quantum-state specific force is typically eight orders of magnitude smaller than the forces that are typically used in charged particle accelerators, but nevertheless suffices to achieve complete control over the motion of polar molecules using techniques akin to those used to control charged particles.

In a Stark decelerator, the longitudinal velocity of a beam of polar molecules is manipulated using an array of longitudinally inhomogeneous electric fields as is shown in Fig. 1. At a given time, the even-numbered stages are switched to high voltage and the odd-numbered stages are grounded. Molecules in low field seeking states that approach the plane of the first electrodes experience

\footnotetext{
${ }^{1}$ Experiments to manipulate the longitudinal motion of molecules in a beam have been considered and tried in the 1950s and 1960s. Electric field deceleration of neutral molecules was first attempted by John King at MIT to produce a slow ammonia beam to obtain a maser with an ultra-narrow linewidth. In the physical chemistry community the experimental efforts of Lennard Wharton, to demonstrate electric field acceleration of a molecular beam, are much better known. At the University of Chicago, he constructed an eleven meter long molecular beam machine for the acceleration of LiF molecules in high-field seeking states from 0.2 to 2.0 $\mathrm{eV}$, aiming to use these high energy beams for reactive scattering studies [10]. Both of these experiments were unsuccessful, and were not continued after the PhD students were finished $[11,12]$. Time-varying electric-field-gradient slowing of Cs atoms has been demonstrated by Harvey Gould and coworkers [13].
} 
the increasing electric field as a potential hill, and will lose kinetic energy on the upward slope of the potential hill. When the molecule leaves the region of high field again, however, it will re-gain the same amount of kinetic energy. The acceleration on the downward slope of the hill can be avoided by switching off the electric field when the molecule has reached a position that is close to the top of the first potential hill. At the same time, the sets of electrodes that were grounded are switched to high voltage. Consequently, the molecule will find itself again in front of a potential hill and will again lose kinetic energy when climbing this hill. When the molecule has reached the high electric field region, the voltages are switched back to the original configuration. By repeating this process many times, the velocity of the molecule can be reduced to an arbitrarily low value.

The amount of kinetic energy that is lost per stage depends on the exact position of the molecule at the time that the fields are being switched. The computer controlled high voltage pulses thus determine the final velocity of the molecule. A second important property of the Stark decelerator is that, like in charged particle accelerators, the deceleration process is phase stable. This means that the process can be described as the trapping of a packet of molecules in a travelling potential well [15]. Hence, the deceleration process does not only work for one molecule in the beam, but it works for all molecules that at the entrance of the decelerator have a longitudinal position and velocity within a certain interval [15-18] (see box 2 for details). It is also required that the molecules keep together in the transverse direction. Molecules in low-field seeking states stay transversely confined to the molecular beam axis as the electric field is always lower on this axis than on the electrodes. Together, these three properties allow one to decelerate (or to accelerate) part of the beam to any desired velocity, while keeping the selected part of the beam together as a compact packet.

The methods described above only work for molecules in a low-field seeking quantum state, and have to date been applied to the molecules $\operatorname{CO}\left(a^{3} \Pi\right)$ [14], $\mathrm{ND}_{3}$ [19], $\mathrm{OH}[20,21], \mathrm{OD}[22], \mathrm{NH}\left(a^{1} \Delta\right)$ [23], $\mathrm{H}_{2} \mathrm{CO}$ [24], and $\mathrm{SO}_{2}$ [25]. High-field seeking molecules are deflected from the molecular beam axis and will eventually be lost. This fundamental problem can be overcome by using alternating gradient $(\mathrm{AG})$ focusers [26, 27], and has been demonstrated for highfield seeking metastable CO molecules [28], ground state YbF molecules [29], and benzonitrile [30].

After exiting the decelerator, the decelerated packets of molecules can be directly used for experiments, or they can be coupled into a variety of elements where they can be manipulated further. Most notably, the molecules can be loaded into traps or into storage rings. The first electrostatic trapping of polar molecules was demonstrated in 2000 using Stark decelerated $\mathrm{ND}_{3}$ molecules [19]. Later, the electrostatic trapping technique was applied to the molecules $\mathrm{OH}[21]$, OD [22], metastable CO [31], and metastable NH [32].

The first electrostatic traps for polar molecules had a quadrupole geometry, as was originally proposed by Wing for Rydberg atoms [33]. Traps with other field geometries have been developed and tested as well. A four elec- 
trode trap geometry that combines a dipole, quadrupole and hexapole field has been tested using decelerated $\mathrm{ND}_{3}$ molecules. By applying different voltages to the electrodes, a double-well or a donut trapping potential can be created [34]. Confinement of Stark decelerated $\mathrm{OH}$ radicals in combined magnetic and electric fields has recently been demonstrated. An electric field was superimposed on a magnetic field to create an overall magnetoelectric trapping potential [35].

Here, the performance of the Stark decelerator and electrostatic quadrupole trap is illustrated using the $\mathrm{OH}\left(X^{2} \Pi_{3 / 2}, J=3 / 2\right)$ radical as a model species. A typical time-of-flight (TOF) profile of $\mathrm{OH}$ radicals exiting the decelerator is shown in Fig. 2(a). The undecelerated part of the beam arrives in the detection region about $3 \mathrm{~ms}$ after its production. In the TOF profile a rich oscillatory structure is observed that results from a modulation of the phase space distribution of the beam in the decelerator [17]. The hole in the beam profile that results from the removal of the packet of molecules that is decelerated, is indicated by the vertical arrow. This packet is split off from the original beam pulse, and arrives in the detection region at later times.

When the packet of molecules is decelerated to near standstill, it can be loaded into the electrostatic quadrupole trap by applying voltages asymmetrically on the three trap electrodes. A potential hill in the trap is created that is higher than the remaining kinetic energy of the molecules. The molecules therefore come to a standstill near the center of the trap. Then, the voltages on the trap electrodes are switched into the "trapping geometry" to create a (nearly) symmetric $500 \mathrm{mK}$ deep potential well. A typical TOF profile that is obtained for OD radicals is shown in Fig. 2(b).

The molecules mainly leave the trap via collisions with particles in the residual gas in the vacuum chamber, or via absorption of blackbody radiation from the (room temperature) surroundings [22].

\section{The Zeeman, Rydberg and optical decelerator}

Inspired in part by the manipulation of polar molecules with electric fields, a magnetic analogue of the Stark decelerator has recently been developed. Deceleration based on the magnetic interaction allows the manipulation of a wide range of atoms and molecules to which the Stark deceleration technique cannot be applied. The required rapid switching of the magnetic fields originally posed a significant experimental challenge. The Zeeman deceleration technique has first been experimentally demonstrated by the deceleration of ground-state $\mathrm{H}$ and D atoms using initially six [36, 37] and later twelve [38] pulsed magnetic field stages; a scheme of the original experimental set-up is shown in Fig. 3. The deceleration stages consist of $7.8 \mathrm{~mm}$ long solenoids of insulated copper wire in which magnetic fields up to $1.5 \mathrm{~T}$ are achieved. The coil design provides a cylindrically symmetric transverse restoring force to the molecular beam axis. When the current through the coils is switched, rise and fall times of the magnetic pulses as short as $5 \mu$ s are achieved. These experiments demonstrate the possibility of switching magnetic fields fast enough to allow deceleration and that 
the deceleration process is subject to phase stability. The Zeeman deceleration technique has also been applied to decelerate metastable Ne atoms [39] in an 18 stage decelerator. Using electromagnetic coils that are encased in magnetic steel shells with Permendur discs, even higher magnetic field densities of $3.6 \mathrm{~T}$ are achieved. Recently, the deceleration of metastable Ne atoms [40] and oxygen molecules [41] to velocities as low as $50 \mathrm{~m} / \mathrm{s}$ using 64 stages has been reported.

Compared to the polar molecules discussed thus far, atoms or molecules in a Rydberg state offer a much larger electric dipole moment. Hence, these particles can be manipulated using only modest electric field strengths in a single, or a few electric field stages. Energy level crossings in the dense Rydberg manifold limit the magnitude of the electric field strengths that can actually be applied. The electric field manipulation of atoms and molecules in high Rydberg states has been pioneered using $\mathrm{H}_{2}$ molecules [42] and Ar atoms [43]. Using a Rydberg decelerator, $\mathrm{H}$ atoms could be stopped and electrostatically trapped in two [44] or three [45] dimensions. The short lifetimes of Rydberg states inherently limit the time that is available to store and study the particles in a trap. If fluorescence to the ground state is the dominant decay process, however, cold samples of ground-state atoms or molecules can be produced via this method. As all atoms and molecules possess Rydberg states, this may provide a general route to cold samples of atoms and molecules.

Optical fields provide another general method to manipulate the motion of neutral particles. An intense optical field will polarize and align molecules [46]. In a laser focus, the polarized molecules will experience a force that is proportional to the gradient of the laser intensity which can be used to focus and trap them. This was experimentally demonstrated by focusing [47] or deflecting [48] a beam of $\mathrm{CS}_{2}$ molecules using a high intensity pulsed laser beam. Optical forces have also been used to reduce the translational energy of molecular beams [49]. Benzene molecules were decelerated from $320 \mathrm{~m} / \mathrm{s}$ to $295 \mathrm{~m} / \mathrm{s}$, while at the same time the xenon carrier gas was decelerated from $320 \mathrm{~m} / \mathrm{s}$ to $310 \mathrm{~m} / \mathrm{s}$, illustrating the generality of this method. Rather than using a single laser beam, much higher forces can be generated by using two near-counterpropagating laser beams as shown in Fig. 3. The two laser beams will interfere to create a socalled optical lattice. Such a lattice forms a periodic array of potential wells for polarizable atoms and molecules. By carefully controlling the frequency difference between the two laser beams, the lattice can be made to move at the same velocity as the molecules in the molecular beam. By slowly lowering the velocity of the lattice, molecules can be decelerated to any given velocity [50]. In this so-called optical Stark decelerator, the chirp of the laser beams needs to be very well controlled, which, in combination with the high intensities, is an experimental challenge. A simpler scheme was recently implemented using a constant frequency offset between the two laser beams such that the lattice moves with a velocity slightly below that of a molecular beam [51]. With a suitable choice of parameters the molecules make exactly a half oscillation within the optical potential. In this way, NO molecules were decelerated from $400 \mathrm{~m} / \mathrm{s}$ to $270 \mathrm{~m} / \mathrm{s}$. 


\section{Applications}

The control over the motion and orientation of molecules by external fields offers a multitude of new possibilities for precision studies of molecular properties and interactions. Over the last few years a number of experiments have been performed that demonstrate this. These experiments can be separated in three distinct classes; beam collision studies, spectroscopic studies, and experiments on molecules that are confined in traps.

Collisions between molecules are highly sensitive to their relative velocity, in particular at low velocities where the kinetic energy of the molecules is comparable to the rotational energy level splitting in the collision complex. At these energies, translational energy can be transferred into rotational energy, effectively binding the molecules transiently together. Long living excitations of the collision complex show up as sharp resonances in the collision energy dependence of the scattering cross sections [52-54]. Stark decelerated molecular beams are well suited for crossed beam scattering experiments, as has been demonstrated recently by scattering a Stark decelerated beam of $\mathrm{OH}$ radicals with a conventional beam of Xe atoms [55]. By varying the collision energy over the energetic thresholds for different scattering channels, the threshold behavior of the inelastic cross sections could be accurately measured. Lower collision energies and a higher energy resolution can be obtained by two Stark decelerated beams crossing at a 90 degree angle. For this, a new crossed-beam scattering machine is currently being built at the Fritz-Haber-Institut. In this machine bi-molecular inelastic or reactive scattering can be studied as a function of the collision energy, with an overall collision energy resolution down to better than $1 \mathrm{~cm}^{-1}$. The combination of the crossed beam machine with a molecular synchrotron (see box 3 for details), in which multiple packets of molecules that revolve around the ring in opposite directions repeatedly interact, offers particularly interesting prospects for molecular collision studies.

Ultimately, the precision in any spectroscopic measurement is limited by the interaction time of the particle to be investigated with the radiation field. In conventional molecular beam experiments this interaction time is typically a few hundred microseconds. The ability to produce slow intense molecular beams enhances significantly the obtainable interaction time and hence resolution. The improved resolution can potentially be used for stringent tests of fundamental physics theories. For instance, polar molecules are being used to test violation of time-reversal symmetry $[56,57]$, in the search for a difference in transition frequency between chiral molecules that are each other's mirror-image [58], and for testing a possible time-variation of the proton-to-electron mass ratio [59, $60]$. Recently, high resolution microwave spectroscopy was performed on Stark decelerated beams of ${ }^{15} \mathrm{ND}_{3}$ [61] and $\mathrm{OH}$ [62] molecules (see Fig. 4). In these proof-of-principle experiments an interaction time of up to a millisecond was obtained. To obtain significantly longer interaction times, a fountain is currently being set up at the Laser Centre Vrije Universiteit Amsterdam. In this fountain, 
molecules are decelerated to a few meters per second, cooled and subsequently launched. The molecules fly upwards some $30 \mathrm{~cm}$ before falling back under gravity, thereby passing a microwave cavity twice. The effective interrogation time in such a Ramsey type measurement scheme includes the entire flight time between the two traversals. In this way an interrogation time of 0.5 seconds can be obtained.

Trapped molecules can be investigated for several seconds. This long interaction time is of limited use to perform high-resolution spectroscopy, as the molecules interact with the trapping field. However, it allows one, for instance, to accurately measure lifetimes of long-lived metastable states. The electrostatic trapping of molecules after Stark deceleration has been used to measure the lifetime of the first vibrationally excited $X^{2} \Pi, v=1$ state of $\mathrm{OH}$, thereby benchmarking the Einstein $A$ coefficients in the important Meinel system of $\mathrm{OH}[63,64]$. The same experimental approach has been applied to accurately measure the lifetimes of $\mathrm{CO}$ molecules in the electronically excited metastable $a^{3} \Pi$ state $[31]$.

The ability to confine molecules in traps also holds great promise in the further development of the field of cold molecules. At sufficiently low temperatures, the de Broglie wavelength of the molecules becomes comparable to, or even larger than, the inter-particle separation. In this regime, quantum degenerate effects dominate the dynamics of the particles, and a Bose-Einstein condensate can be formed. The anisotropic long-range dipole-dipole interaction is predicted to give rise to new and rich physics in these cold dipolar gases [65-67], as has already been observed in a Bose-Einstein condensate of Chromium atoms with strong magnetic dipole-dipole interactions [68, 69]. The strong electric dipoledipole interactions offers also interesting prospects in quantum computation schemes $[70,71]$.

To experimentally study these phenomena, the temperature of the trapped sample of molecules needs to be further reduced. Various cooling schemes have been proposed to achieve temperatures below $1 \mathrm{mK}$. The most promising schemes are evaporative cooling, in which the molecules re-thermalize after selective removal of the hottest molecules, and sympathetic cooling, in which the molecules are brought into contact with an ultracold atomic gas and equilibrate with it via elastic collisions. Another cooling scheme that is actively being pursued is cavity-assisted laser cooling [72-75]. Trap loss due to inelastic collisions, that transfer the molecules from the trapped low-field seeking state to an anti-trapped high-field seeking state, is expected to be severe in the first two cooling schemes. It is therefore essential to confine molecules in high-field seeking states, eliminating the inelastic loss channels. An AC trap, in which a saddle-point electric field geometry is rotated fast enough to create a time-averaged potential well for high-field seeking states, has already been demonstrated for $\mathrm{ND}_{3}$ molecules [76, 77]. 


\section{Conclusions}

The different tools that were developed in the past to transversally manipulate molecular beams, dating back to the Stern and Rabi era, have proven to be crucial for developments beyond molecular physics alone. The merging of molecular beam methods with those of accelerator physics now provides new tools to achieve complete control over the full three-dimensional motion of molecules. Over the last few years decelerators, lenses, bunchers, traps, storage rings and a synchrotron for neutral molecules have been demonstrated. Intense molecular beams with a tunable velocity and with a tunable width of the velocity distribution can now be produced. This new molecular beam technology is expected to become a valuable tool in a variety of chemical physics experiments, adding a new dimension to the long and rich history of the manipulation of molecular beams with external fields.

\section{Acknowledgements}

The experiments that are described in this Review and that have been performed in our laboratory are the result of almost ten years of research by a large group of people. We are greatly indebted to the students, postdocs, senior scientists and research technicians that have been involved in this work, and without whom these experiments would not have been possible. The measurements shown in Figure 2(a) were performed with the help of S. Hoekstra, J.J. Gilijamse, and J. Küpper. H.L. Bethlem acknowledges financial support from the Netherlands Organization for Scientific Research (NWO) via a VENI-grant. The fruitful collaboration and the open exchange of ideas with the other laboratories working in this area are greatly appreciated.

$<<<$ begin box 1 "molecular beams" $>>>$

A molecular beam is formed by letting a gas pass from a high-pressure container through a small orifice into an evacuated chamber, i.e., by a controlled leak. If the hole is much smaller than the mean free path of the gas, molecules will every now and then escape from the container through the hole without undergoing collisions. In this case, the velocity distribution as well as the distribution over the internal degrees of freedom of the molecules (rotation, vibration) in the beam is the same as that in the container. These beams are said to be effusive. Although the most probable velocity of molecules in an effusive beam originating from a room-temperature container is a few hundred meters per second, a small fraction of the molecules will have velocities below $30 \mathrm{~m} / \mathrm{s}$. Using either curved electrostatic guides [78-81] or a mechanical selector - a series of rotating blades [82] - these slow molecules can be filtered out of the effusive beam and used for experiments [83]. Higher fluxes of slow and internally colder molecules can be obtained when the effusive beam is extracted from a cryogenic container $[84,85]$.

If the pressure in the container is raised or if the size of the hole is increased, 
the mean free path of the gas becomes smaller than the orifice. In this case, molecules escaping through the hole collide frequently, and adiabatic cooling of all degrees of freedom takes place in the expansion region. The total energy available per molecule in the gas in the container is converted into kinetic energy (directed flow), leading to supersonic beams of internally cold molecules [86]. The terminal temperature in the beam is limited by the formation of clusters, which is reduced when the molecule of interest is expanded in a dilute mixture with a noble gas. When these beams are operated in a pulsed mode, pumping requirements are less severe and mm-sized orifices can be used. In these pulsed beams densities of $10^{13}$ molecules $/ \mathrm{cm}^{3}$ at translational temperatures below $1 \mathrm{~K}$ can be obtained. The rotational temperature in the beam is normally close to the translational temperature, whereas the vibrational degrees of freedom are known to cool considerably less well. The velocity of the beam is dictated by the mass of the carrier gas and by the temperature and pressure of the source [87]. Molecules seeded in a room-temperature $\mathrm{Kr}(\mathrm{Xe})$ expansion move at a terminal velocity of about $440 \mathrm{~m} / \mathrm{s}(330 \mathrm{~m} / \mathrm{s})$. These seeded pulsed beams are ideal starting points for Stark, Zeeman, and optical deceleration experiments.

Rather than grabbing with fields onto the molecules, other techniques to produce slow molecular beams have been developed. In a crossed or counter propagating beam setup, the kinetic energy transfer in collisions [88] or reactions [89] between molecules can be exploited to produce slow or stationary molecules. Molecules can also be slowed and loaded into a magnetic trap by introducing a molecular beam into a cryogenic buffer gas [90]. Mechanical methods, originally pioneered by Moon and coworkers in the 70's to accelerate a molecular beam [91], have been developed as well. For instance, the laboratory velocity of a molecular beam can be lowered by a nozzle that is mounted on a back spinning rotor [92, 93] or by elastic reflection from a receding substrate [94].

$<<<$ end box 1 "molecular beams" $>>>$

$<<<$ begin box 2 "phase stability" $>>>$

In analogy to the operation of linear accelerators for charged particles, the concept of phase stability is essential to the operation of Stark, Zeeman and optical decelerators. Phase stability is the reason that these decelerators actually work. It is discussed in more detail below for a Stark decelerator.

In a Stark decelerator, the voltages that are applied to the electric field stages are switched such that they are synchronous with a hypothetical molecule, aptly referred to as the 'synchronous' molecule, flying on the molecular beam axis. Molecules that are slightly ahead of the synchronous molecule will be decelerated more than the synchronous molecule, while molecules that are slightly behind the synchronous molecule will be decelerated less. As a consequence, molecules with a slightly different longitudinal position and velocity from that of the synchronous molecule experience a force towards the synchronous molecule and will oscillate around it. The position of the synchronous molecule with respect to the electrodes at high voltage as the field are being switched - how far the molecule has entered the field - is expressed in terms of a 'phase angle', $\phi$. When this phase is close to $90^{\circ}$, the deceleration per stage is large but the longitudinal velocity and position spread of the molecules that will be accepted in the 
deceleration process is small. When $\phi$ is small the acceptance is large but the deceleration per stage is small [16].

In the transverse direction, molecules experience a restoring force towards the beam axis because the electric fields are higher at the electrodes than on the molecular beam axis. This focusing force strongly depends on the exact position of the molecule along the molecular beam axis. Close to the electrodes at high voltage the transverse focusing is strong while the transverse focusing is small close to the electrodes at ground potential. The oscillatory motion of molecules around the synchronous molecule results in a time-dependent transverse force that can result in unstable trajectories through the decelerator [95].

In Fig. 6 the six dimensional acceptance, i.e., the product of the position and velocity spread of molecules in each direction that is accepted by the decelerator, is shown as a function of the phase angle for two different operation modes of the decelerator. The acceptance that is obtained from numerical trajectory simulations taking the true force on the molecules into account is given as data points, that are connected by straight line segments. The acceptance that results from the combined model for the longitudinal [16] and transverse [95] motion of molecules in a Stark decelerator, that neglects any coupling between the motion in each orthogonal direction, is given by the smooth curves. In the conventional operation mode of a Stark decelerator, referred to as $s=1$ [17], each deceleration stage is used to remove kinetic energy from the molecules. It is seen that the actual acceptance of the decelerator is much lower than the model prediction, in particular for phase angles below $70^{\circ}$; the instabilities that result from the coupling between the longitudinal and transverse motion are severe, and limit the efficiency of present Stark decelerators. The motions can be uncoupled by using only every third stage for deceleration, while the intermediate stages are used for transverse focussing. The performance of the decelerator in this so-called $s=3$ mode [17] follows the model prediction much better, and a significantly higher phase space acceptance can be achieved.

In Stark and Zeeman decelerators, phase stability is ensured by repeatedly switching between two different static configurations, thereby confining the molecules in an effective travelling potential well. This is fundamentally different from the situation in the optical Stark decelerator, where genuine travelling wells are present. The trapping of polar molecules in genuine travelling potential wells closely above a microstructured electrode array has recently been demonstrated [96].

$$
<<<\text { end box } 2 \text { "phase stability" }>>>
$$

\section{$<<<$ begin box 3 "synchrotron" $>>>$}

Rather than confining molecules in a potential energy minimum at a single location in space as is done in a trap, molecules can also be confined in a minimum along a circle. The advantage of such a storage ring over a trap is that packets of particles with a non-zero mean velocity can be confined. While revolving in the ring, these particles can be made to interact repeatedly, at well defined times and at distinct positions with electromagnetic fields and/or other 
particles.

A simple storage ring for polar molecules can be devised by bending an electrostatic hexapole into a torus. In the first experiments with such a prototype storage ring, beams of ammonia molecules were decelerated to a velocity of $92 \mathrm{~m} / \mathrm{s}$, bunched [97], and tangentially injected into the ring [98, 99], in which up to 50 distinct round trips could be observed. As a result of the longitudinal velocity spread, the packet of molecules gradually spreads out along the ring upon making successive round trips, until they fill the entire ring. In order to counteract this spreading a storage ring consisting of two half-rings separated by a $2 \mathrm{~mm}$ gap was constructed, as is schematically shown in the top part of Fig. 7 [100]. By appropriately switching the voltages applied to the electrodes as the molecules pass through the gaps between the two half-rings, molecules experience a force that keeps them together in a compact bunch. This structure is the neutral analog of a synchrotron for charged particles. The lower part of Fig. 7 shows the density of molecules stored in the ring as a function of storage time. It is seen that the width of the stored packet stays approximately constant. Bunching ensures a high density of stored molecules, in addition it makes it possible to inject multiple - either co-linear or counter propagating - packets into the ring without affecting the packet(s) already stored. At the Fritz-Haber-Institut, we are currently developing a synchrotron consisting of 40 segments. This synchrotron is designed to simultaneously confine 20 packets of molecules flying clockwise and counter-clockwise.

$<<<$ end box 3 "synchrotron" $>>>$

\section{References}

[1] Scoles, G., editor. Atomic and molecular beam methods, volume 1 \& 2. Oxford University Press, New York, NY, USA, (1988 \& 1992).

[2] Gerlach, W. and Stern, O. The experimental evidence of direction quantisation in the magnetic field. Zeitschrift Für Physik 9, 349-352 (1922).

[3] Rabi, I. I., Millman, S., Kusch, P., and Zacharias, J. P. The molecular beam resonance method for measuring nuclear magnetic moments. Phys. Rev. 55, 526-535 (1939).

[4] Friedburg, H. and Paul, W. Optische Abbildung mit neutralen Atomen. Die Naturwissenschaften 38, 159-160 (1951).

[5] Bennewitz, H. and Paul, W. Eine Methode zur Bestimmung von Kernmomenten mit fokussiertem Atomstrahl. Z. Phys. 139, 489-497 (1954).

[6] Bennewitz, H. G., Paul, W., and Schlier, C. Fokussierung polarer Moleküle. Z. Phys. 141, 6-15 (1955).

[7] Gordon, J. P., Zeiger, H. J., and Townes, C. H. Molecular microwave oscillator and new hyperfine structure in the microwave spectrum of $\mathrm{NH}_{3}$. Phys. Rev. 95, 282-284 (1954). 
[8] Gordon, J. P., Zeiger, H. J., and Townes, C. H. The maser - New type of microwave amplifier, frequency standard, and spectrometer. Phys. Rev. 99, 1264-1274 (1955).

[9] Stolte, S. Reactive scattering studies on oriented molecules. Berichte der Bunsen Gesellschaft-Phys. Chem. Chem. Phys. 86, 413-421 (1982).

[10] Wolfgang, R. Chemical accelerators. Sci. Am. 219, 44 (1968).

[11] Golub, R. On decelerating molecules. PhD thesis, MIT, Cambridge, USA, (1967).

[12] Bromberg, E. E. A. Acceleration and alternate-gradient focusing of neutral polar diatomic molecules. PhD thesis, University of Chicago, USA, (1972).

[13] Maddi, J. A., Dinneen, T. P., and Gould, H. Slowing and cooling molecules and neutral atoms by time-varying electric-field gradients. Phys. Rev. A 60, 3882-3891 (1999).

[14] Bethlem, H. L., Berden, G., and Meijer, G. Decelerating neutral dipolar molecules. Phys. Rev. Lett. 83, 1558-1561 (1999).

[15] Bethlem, H. L., Berden, G., van Roij, A. J. A., Crompvoets, F. M. H., and Meijer, G. Trapping neutral molecules in a traveling potential well. Phys. Rev. Lett. 84, 5744-5747 (2000).

[16] Bethlem, H. L., Crompvoets, F. M. H., Jongma, R. T., van de Meerakker, S. Y. T., and Meijer, G. Deceleration and trapping of ammonia using time-varying electric fields. Phys. Rev. A 65, 053416 (2002).

[17] van de Meerakker, S. Y. T., Vanhaecke, N., Bethlem, H. L., and Meijer, G. Higher-order resonances in a Stark decelerator. Phys. Rev. A 71, 053409 (2005).

[18] Gubbels, K., Meijer, G., and Friedrich, B. Analytic wave model of Stark deceleration dynamics. Phys. Rev. A 73, 063406 (2006).

[19] Bethlem, H. L., Berden, G., Crompvoets, F. M. H., Jongma, R. T., van Roij, A. J. A., and Meijer, G. Electrostatic trapping of ammonia molecules. Nature 406, 491-494 (2000).

[20] Bochinski, J. R., Hudson, E. R., Lewandowski, H. J., Meijer, G., and Ye, J. Phase space manipulation of cold free radical $\mathrm{OH}$ molecules. Phys. Rev. Lett. 91, 243001 (2003).

[21] van de Meerakker, S. Y. T., Smeets, P. H. M., Vanhaecke, N., Jongma, R. T., and Meijer, G. Deceleration and electrostatic trapping of OH radicals. Phys. Rev. Lett. 94, 023004 (2005). 
[22] Hoekstra, S., Gilijamse, J. J., Sartakov, B., Vanhaecke, N., Scharfenberg, L., van de Meerakker, S. Y. T., and Meijer, G. Optical pumping of trapped neutral molecules by blackbody radiation. Phys. Rev. Lett. 98, 133001 (2007).

[23] van de Meerakker, S. Y. T., Labazan, I., Hoekstra, S., Küpper, J., and Meijer, G. Production and deceleration of a pulsed beam of metastable NH $\left(a^{1} \Delta\right)$ radicals. J. Phys. B 39, S1077-S1084 (2006).

[24] Hudson, E. R., Ticknor, C., Sawyer, B. C., Taatjes, C. A., Lewandowski, H. J., Bochinski, J. R., Bohn, J. L., and Ye, J. Production of cold formaldehyde molecules for study and control of chemical reaction dynamics with hydroxyl radicals. Phys. Rev. A 73, 063404 (2006).

[25] Jung, S., Tiemann, E., and Lisdat, Ch. Cold atoms and molecules from fragmentation of decelerated $\mathrm{SO}_{2}$. Phys. Rev. A 74, 040701 (2006).

[26] Auerbach, D., Bromberg, E. E. A., and Wharton, L. Alternate-gradient focusing of molecular beams. J. Chem. Phys. 45, 2160-2166 (1966).

[27] Bethlem, H. L., Tarbutt, M. R., Küpper, J., Carty, D., Wohlfart, K., Hinds, E. A., and Meijer, G. Alternating gradient focusing and deceleration of polar molecules. J. Phys. B 39, R263-R291 (2006).

[28] Bethlem, H. L., van Roij, A. J. A., Jongma, R. T., and Meijer, G. Alternate gradient focusing and deceleration of a molecular beam. Phys. Rev. Lett. 88, 133003 (2002).

[29] Tarbutt, M. R., Bethlem, H. L., Hudson, J. J., Ryabov, V. L., Ryzhov, V. A., Sauer, B. E., Meijer, G., and Hinds, E. A. Slowing heavy, groundstate molecules using an alternating gradient decelerator. Phys. Rev. Lett. 92, 173002 (2004).

[30] Wohlfart, K., Grätz, F., Filsinger, F., Haak, H., Meijer, G., and Küpper, J. Alternating-gradient focusing and deceleration of large molecules. Phys. Rev. A 77, 031404 (2008).

[31] Gilijamse, J. J., Hoekstra, S., Meek, S. A., Metsälä, M., van de Meerakker, S. Y. T., Meijer, G., and Groenenboom, G. C. The radiative lifetime of metastable CO $\left(a^{3} \Pi, v=0\right)$. J. Chem. Phys. 127, 221102 (2007).

[32] Hoekstra, S., Metsälä, M., Zieger, P. C., Scharfenberg, L., Gilijamse, J. J., Meijer, G., and van de Meerakker, S. Y. T. Electrostatic trapping of metastable NH molecules. Phys. Rev. A 76, 063408 (2007).

[33] Wing, W. H. Electrostatic trapping of neutral atomic particles. Phys. Rev. Lett. 45, 631-634 (1980).

[34] van Veldhoven, J., Bethlem, H. L., Schnell, M., and Meijer, G. Versatile electrostatic trap. Phys. Rev. A 73, 063408 (2006). 
[35] Sawyer, B. C., Lev, B. L., Hudson, E. R., Stuhl, B. K., Lara, M., Bohn, J. L., and Ye, J. Magnetoelectrostatic trapping of ground state $\mathrm{OH}$ molecules. Phys. Rev. Lett. 98, 253002 (2007).

[36] Vanhaecke, N., Meier, U., Andrist, M., Meier, B. H., and Merkt, F. Multistage Zeeman deceleration of hydrogen atoms. Phys. Rev. A 75, 031402 (2007).

[37] Hogan, S. D., Sprecher, D., Andrist, M., Vanhaecke, N., and Merkt, F. Zeeman deceleration of $\mathrm{H}$ and D. Phys. Rev. A 76, 023412 (2007).

[38] Hogan, S. D., Wiederkehr, A. W., Andrist, M., Schmutz, H., and Merkt, F. Slow beams of atomic hydrogen by multistage Zeeman deceleration. $J$. Phys. B: At. Mol. Opt. Phys. 41, 081005 (2008).

[39] Narevicius, E., Parthey, C. G., Libson, A., Narevicius, J., Chavez, I., Even, U., and Raizen, M. G. An atomic coilgun: using pulsed magnetic fields to slow a supersonic beam. New J. of Physics 9, 358 (2007).

[40] Narevicius, E., Libson, A., Parthey, C. G., Chavez, I., Narevicius, J., Even, U., and Raizen, M. G. Stopping supersonic beams with a series of pulsed electromagnetic coils: an atomic coilgun. Phys. Rev. Lett. 100, 093003 (2008).

[41] Narevicius, E., Libson, A., Parthey, C. G., Chavez, I., Narevicius, J., Even, U., and Raizen, M. G. Stopping supersonic oxygen with a series of pulsed electromagnetic coils: A molecular coilgun. arXiv:0804.0219v1 (2008).

[42] Yamakita, Y., Procter, S. R., Goodgame, A. L., Softley, T. P., and Merkt, F. Deflection and deceleration of hydrogen Rydberg molecules in inhomogeneous electric fields. J. Chem. Phys. 121, 1419-1431 (2004).

[43] Vliegen, E., Wörner, H. J., Softley, T. P., and Merkt, F. Nonhydrogenic effects in the deceleration of Rydberg atoms in inhomogeneous electric fields. Phys. Rev. Lett. 92, 033005 (2004).

[44] Vliegen, E., Hogan, S. D., Schmutz, H., and Merkt, F. Stark deceleration and trapping of hydrogen Rydberg atoms. Phys. Rev. A 76, 023405 (2007).

[45] Hogan, S. D. and Merkt, F. Demonstration of three-dimensional electrostatic trapping of state-selected Rydberg atoms. Phys. Rev. Lett. 100, 043001 (2008).

[46] Friedrich, B. and Herschbach, D. Alignment and trapping of molecules in intense laser fields. Phys. Rev. Lett. 74, 4623-4626 (1995).

[47] Zhao, B. S., Sung Chung, H., Cho, K., Hyup Lee, S., Hwang, S., Yu, J., Ahn, Y. H., Sohn, J. Y., Kim, D. S., Kyung Kang, W., and Chung, D. S. Molecular lens of the nonresonant dipole force. Phys. Rev. Lett. 85, 27052708 (2000). 
[48] Stapelfeldt, H., Sakai, H., Constant, E., and Corkum, P. B. Deflection of neutral molecules using the nonresonant dipole force. Phys. Rev. Lett. 79, 2787-2790 (1997).

[49] Fulton, R., Bishop, A. I., and Barker, P. F. Optical Stark decelerator for molecules. Phys. Rev. Lett. 93, 243004 (2004).

[50] Barker, P. F. and Shneider, M. N. Slowing molecules by optical microlinear deceleration. Phys. Rev. A 66, 065402 (2002).

[51] Fulton, R., Bishop, A. I., Shneider, M. N., and Barker, P. F. Controlling the motion of cold molecules with deep periodic optical potentials. Nature Physics 2, 465-468 (2006).

[52] Balakrishnan, N. and Dalgarno, A. Vibrational relaxation of CO collisions with ${ }^{4} \mathrm{He}$ at ultracold temperatures. J. Chem. Phys. 113, 621-627 (2000).

[53] Hutson, J. and Soldan, P. Molecule formation in ultracold atomic gases. Int. Rev. Phys. Chem. 25, 497-526 (2006).

[54] Bodo, E. and Gianturco, F. A. Collisional quenching of molecular rovibrational energy by He buffer loading at ultralow energies. Int. Rev. Phys. Chem. 25, 313-351 (2006).

[55] Gilijamse, J. J., Hoekstra, S., van de Meerakker, S. Y. T., Groenenboom, G. C., and Meijer, G. Near-threshold inelastic collisions using molecular beams with a tunable velocity. Science 313, 1617-1620 (2006).

[56] Hudson, J. J., Sauer, B. E., Tarbutt, M. R., and Hinds, E. A. Measurement of the electron electric dipole moment using $\mathrm{YbF}$ molecules. Phys. Rev. Lett. 89, 023003 (2002).

[57] Kawall, D., Bay, F., Bickman, S., Jiang, Y., and DeMille, D. Precision Zeeman-Stark spectroscopy of the metastable $a(1)\left[{ }^{3} \Sigma^{+}\right]$state of PbO. Phys. Rev. Lett. 92, 133007 (2004).

[58] Daussy, C., Marrel, T., Amy-Klein, A., Nguyen, C. T., Bordé, Ch. J., and Chardonnet, Ch. Limit on the parity nonconserving energy difference between the enantiomers of a chiral molecule by laser spectroscopy. Phys. Rev. Lett. 83, 1554-1557 (1999).

[59] Reinhold, E., Buning, R., Hollenstein, U., Ivanchik, A., Petitjean, P., and Ubachs, W. Indication of a cosmological variation of the proton-electron mass ratio based on laboratory measurement and reanalysis of $\mathrm{H}_{2}$ spectra. Phys. Rev. Lett. 96, 151101 (2006).

[60] Shelkovnikov, A., Butcher, R. J., Chardonnet, Ch., and Amy-Klein, A. Stability of the proton-to-electron mass ratio. Phys. Rev. Lett. 100, 150801 (2008). 
[61] van Veldhoven, J., Küpper, J., Bethlem, H. L., Sartakov, B., van Roij, A. J. A., and Meijer, G. Decelerated molecular beams for high-resolution spectroscopy: The hyperfine structure of ${ }^{15} \mathrm{ND}_{3}$. Eur. Phys. J. D 31, 337349 (2004).

[62] Hudson, E. R., Lewandowski, H. J., Sawyer, B. C., and Ye, J. Cold molecule spectroscopy for constraining the evolution of the fine structure constant. Phys. Rev. Lett. 96, 143004 (2006).

[63] van de Meerakker, S. Y. T., Vanhaecke, N., van der Loo, M. P. J., Groenenboom, G. C., and Meijer, G. Direct measurement of the radiative lifetime of vibrationally excited OH radicals. Phys. Rev. Lett. 95, 013003 (2005).

[64] van der Loo, M. P. J. and Groenenboom, G. C. Theoretical transition probabilities for the $\mathrm{OH}$ Meinel system. J. Chem. Phys. 126, 114314 (2007).

[65] Santos, L., Shlyapnikov, G. V., Zoller, P., and Lewenstein, M. BoseEinstein condensation in trapped dipolar gases. Phys. Rev. Lett. 85, 17911794 (2000).

[66] Baranov, M., Dobrek, L., Góral, K., Santos, L., and Lewenstein, M. Ultracold dipolar gases - a challenge for experiments and theory. Phys. Scr. T102, 74-81 (2002).

[67] Krems, R. V. Molecules near absolute zero and external field control of atomic and molecular dynamics. Int. Rev. Phys. Chem. 24, 99-118 (2005).

[68] Lahaye, T., Koch, T., Fröhlich, B., Fattori, M., Metz, J., Griesmaier, A., Giovanazzi, S., and Pfau, T. Strong dipolar effects in a quantum ferrofluid. Nature 448, 672-675 (2007).

[69] Koch, T., Lahaye, T., Metz, J., Fröhlich, B., Griesmaier, A., and Pfau, T. Stabilization of a purely dipolar quantum gas against collapse. Nature Physics 4, 218-222 (2008).

[70] DeMille, D. Quantum computation with trapped polar molecules. Phys. Rev. Lett. 88, 067901 (2002).

[71] André, A., DeMille, D., Doyle, J. M., Lukin, M. D., Maxwell, S. E., Rabl, P., Schoelkopf, R. J., and Zoller, P. A coherent all-electrical interface between polar molecules and mesoscopic superconducting resonators. Nature physics 2, 636-642 (2006).

[72] Vuletić, V. and Chu, S. Laser cooling of atoms, ions, or molecules by coherent scattering. Phys. Rev. Lett. 84, 3787-3790 (2000).

[73] Domokos, P. and Ritsch, H. Mechanical effects of light in optical resonators. J. Opt. Soc. Am. B 20, 1098-1130 (2003). 
[74] Morigi, G., Pinkse, P. W. H., Kowalewski, M., and de Vivie-Riedle, R. Cavity cooling of internal molecular motion. Phys. Rev. Lett. 99, 073001 (2007).

[75] Lev, B. L., Vukics, A., Hudson, E. R., Sawyer, B. C., Domokos, P., Ritsch, H., and Ye, J. Prospects for the cavity-assisted laser cooling of molecules. Phys. Rev. A 77, 023402 (2008).

[76] van Veldhoven, J., Bethlem, H. L., and Meijer, G. AC electric trap for ground-state molecules. Phys. Rev. Lett. 94, 083001 (2005).

[77] Bethlem, H. L., van Veldhoven, J., Schnell, M., and Meijer, G. Trapping polar molecules in an ac trap. Phys. Rev. A 74(6), 063403 (2006).

[78] Rangwala, S. A., Junglen, T., Rieger, T., Pinkse, P. W. H., and Rempe, G. Continuous source of translationally cold dipolar molecules. Phys. Rev. A 67, 043406 (2003).

[79] Rieger, T., Junglen, T., Rangwala, S. A., Pinkse, P. W. H., and Rempe, G. Continuous loading of an electrostatic trap for polar molecules. Phys. Rev. Lett. 95, 173002 (2005).

[80] Junglen, T., Rieger, T., Pinkse, P. W. H., and Rempe, G. Two-dimensional trapping of dipolar molecules in time-varying electric fields. Phys. Rev. Lett. 92, 223001 (2004).

[81] Tsuji, H., Okuda, Y., Sekiguchi, T., and Kanamori, H. Velocity distribution of the pulsed $\mathrm{ND}_{3}$ molecular beam selected by a quadrupole Stark velocity filter. Chem. Phys. Lett. 436, 331-334 (2008).

[82] Deachapunya, S., Fagan, P. J., Major, A. G., Reiger, E., Ritsch, H., Stefanov, A., Ulbricht, H., and Arndt, M. Slow beams of massive molecules. Eur. Phys. J. D 46, 307-313 (2008).

[83] Willitsch, S., Bell, M. T., Gingell, A. D., Procter, S. R., and Softley, T. P. Cold reactive collisions between laser-cooled ions and velocity-selected neutral molecules. Phys. Rev. Lett. 100, 043203 (2008).

[84] Patterson, D. and Doyle, J. M. Bright, guided molecular beam with hydrodynamic enhancement. J. Chem. Phys. 126, 154307 (2007).

[85] Maxwell, S. E., Brahms, N., deCarvalho, R., Glenn, D. R., Helton, J. S., Nguyen, S. V., Patterson, D., Petricka, J., DeMille, D., and Doyle, J. M. High-flux beam source for cold, slow atoms or molecules. Phys. Rev. Lett. 95(17), 173201 (2005).

[86] Levy, D. The spectroscopy of very cold gases. Science 214, 263-269 (1981).

[87] Christen, W. and Rademann, K. Cooling and slowing in high-pressure jet expansions. Phys. Rev. A 77, 012702 (2008). 
[88] Elioff, M. S., Valentini, J. J., and Chandler, D. W. Subkelvin cooling NO molecules via "billiard-like" collisions with argon. Science 302, 1940-1943 (2003).

[89] Liu, N.-N. and Loesch, H. Kinematic slowing of molecules formed by reactive collisions. Phys. Rev. Lett. 98, 103002 (2007).

[90] Campbell, W. C., Tsikata, E., Lu, H.-I., van Buuren, L. D., and Doyle, J. M. Magnetic trapping and Zeeman relaxation of $\mathrm{NH} X^{3} \Sigma^{-}$. Phys. Rev. Lett. 98, 213001 (2007).

[91] Moon, P. B., Rettner, C. T., and Simons, J. P. Rotor accelerated molecular beams. J. of the Chem. Soc., Faraday Trans. II 74, 630-643 (1978).

[92] Gupta, M. and Herschbach, D. A mechanical means to produce intense beams of slow molecules. J. Phys. Chem. A 103, 10670-10673 (1999).

[93] Gupta, M. and Herschbach, D. Slowing and speeding molecular beams by means of a rapidly rotating source. J. Phys. Chem. A 105, 1626-1637 (2001).

[94] Narevicius, E., Libson, A., Riedel, M. F., Parthey, C. G., Chavez, I., Even, U., and Raizen, M. G. Coherent slowing of a supersonic beam with an atomic paddle. Phys. Rev. Lett. 98, 103201 (2007).

[95] van de Meerakker, S. Y. T., Vanhaecke, N., Bethlem, H. L., and Meijer, G. Transverse stability in a Stark decelerator. Phys. Rev. A 73, 023401 (2006).

[96] Meek, S. A., Bethlem, H. L., Conrad, H., and Meijer, G. Trapping molecules on a chip in traveling potential wells. Phys. Rev. Lett. 100, 153003 (2008).

[97] Crompvoets, F. M. H., Jongma, R. T., Bethlem, H. L., van Roij, A. J. A., and Meijer, G. Longtudinal focusing and cooling of a molecular beam. Phys. Rev. Lett. 89, 093004, August (2002).

[98] Crompvoets, F. M. H., Bethlem, H. L., Jongma, R. T., and Meijer, G. A prototype storage ring for neutral molecules. Nature 411, 174-176 (2001).

[99] Crompvoets, F. M. H., Bethlem, H. L., Küpper, J., van Roij, A. J. A., and Meijer, G. Dynamics of neutral molecules stored in a ring. Phys. Rev. A 69, 063406 (2004).

[100] Heiner, C. E., Carty, D., Meijer, G., and Bethlem, H. L. A molecular synchrotron. Nature Physics 3, 115-118 (2007). 
Figure 1: Scheme of the experimental set-up. A pulsed molecular beam is produced and passes through a skimmer, hexapole and Stark decelerator into the detection region. The electric field stages of the decelerator are composed of two opposing electrodes that are connected to power supplies of opposite polarity. Alternating rods are connected to each other. The potential energy $W(z)$ of a molecule that is in a low-field seeking quantum state, i.e. a molecule that gains Stark energy when flying into an increasing electric field, is shown as a function of the position $z$ along the beam axis. A photograph of one of the decelerators that is operational in our laboratory is shown in the top inset.

Figure 2: Decelerated and trapped hydroxyl radicals. (a) Observed TOF profile of a molecular beam of $\mathrm{OH}$ radicals in the $X^{2} \Pi_{3 / 2}(v=0, J=3 / 2, f, F=2)$ level exiting the Stark decelerator. The molecular beam has a mean velocity of $370 \mathrm{~m} / \mathrm{s}$ and the decelerator is programmed to slow down a packet of molecules from $370 \mathrm{~m} / \mathrm{s}$ to $100 \mathrm{~m} / \mathrm{s}$. (b) Observed TOF profile when a packet of OD molecules is slowed down to standstill and confined in an electrostatic quadrupole trap. In the inset, the signal of the trapped OD radicals is shown on a 15 second timescale. (Partially reproduced from S. Hoekstra et al. [22] with permission). 
Figure 3: Scheme of the Zeeman and optical Stark decelerator. (a) The Zeeman decelerator as used by Vanhaecke et al. [36] consisting of six solenoids of insulated copper wire. The decelerator was used to slow down seeded beams of $\mathrm{H}$ and D atoms. (b) The optical Stark decelerator as used by Fulton et al. [51]. An optical lattice is created by crossing two intense pulsed laser beams. The two laser beams have a slightly different frequency, such that the optical lattice has a velocity that is matched to that of the molecular beam. The molecules are detected by ionizing them using a third laser beam.

Figure 4: High resolution spectroscopy using Stark decelerated beams. (a) Schematic of a microwave spectroscopy experiment at JILA in which a beam of $\mathrm{OH}$ radicals is Stark decelerated to $200 \mathrm{~m} / \mathrm{s}$, and interrogated in a $10 \mathrm{~cm}$ long microwave cavity. (b) Ramsey microwave spectroscopy for the transition between the $F=2$ hyperfine states of the two $\Lambda$-doublet components of the rotational ground state of the $\mathrm{OH}$ radical. (Reproduced from Hudson et al. [62] with permission).

Figure 5: Schematic view of a supersonic expansion. A high-pressure gas is expanded through a small hole into vacuum. The inset shows the velocity distribution of room temperature ammonia molecules in the container and in the supersonic beam. By using a noble gas as carrier the velocity distribution is further narrowed. The terminal velocity of the ammonia molecules will then be the same as that of the carrier gas.

Figure 6: The six dimensional phase space acceptance of a Stark decelerator. The acceptance that results from numerical trajectory simulations is shown as a function of the phase angle (squares for $s=1$ and triangles for $s=3$ ), together with the model prediction (dashed line for $s=1$ and solid line for $s=3$ ). The simulations are performed for $\mathrm{OH}$ radicals in the $X^{2} \Pi_{3 / 2}, J=$ $3 / 2, M \Omega=-9 / 4, f$ state, that propagate through a Stark decelerator of variable length. The decelerator is operated with $40 \mathrm{kV}$ voltage difference on opposing electrodes. The electrodes have a diameter of $4.50 \mathrm{~mm}$, are placed at a center to center distance $L=8.25 \mathrm{~mm}$ from each other, and leave a $3 \times 3 \mathrm{~mm}^{2}$ area around the beam axis. For each simulation, the length of the decelerator is chosen to slow down the synchronous molecule from $450 \mathrm{~m} / \mathrm{s}$ to $150 \mathrm{~m} / \mathrm{s}$, to exclude loss of molecules due to excessive transverse focussing at low velocities. This is expected below $50 \mathrm{~m} / \mathrm{s}$ for $s=1$ and below $150 \mathrm{~m} / \mathrm{s}$ for $s=3$.

Figure 7: The molecular synchrotron. (a) The synchrotron consists of two hexapole half-rings with a $12.5 \mathrm{~cm}$ radius separated by a $2 \mathrm{~mm}$ gap. (b) Density of ammonia molecules at the detection zone inside the synchrotron as a function of storage time up to the $40^{\text {th }}$ round trip. Expanded views of two TOF profiles are shown as insets, more clearly illustrating the absolute widths of these peaks. (Reproduced from C.E. Heiner et al. [100] with permission). 

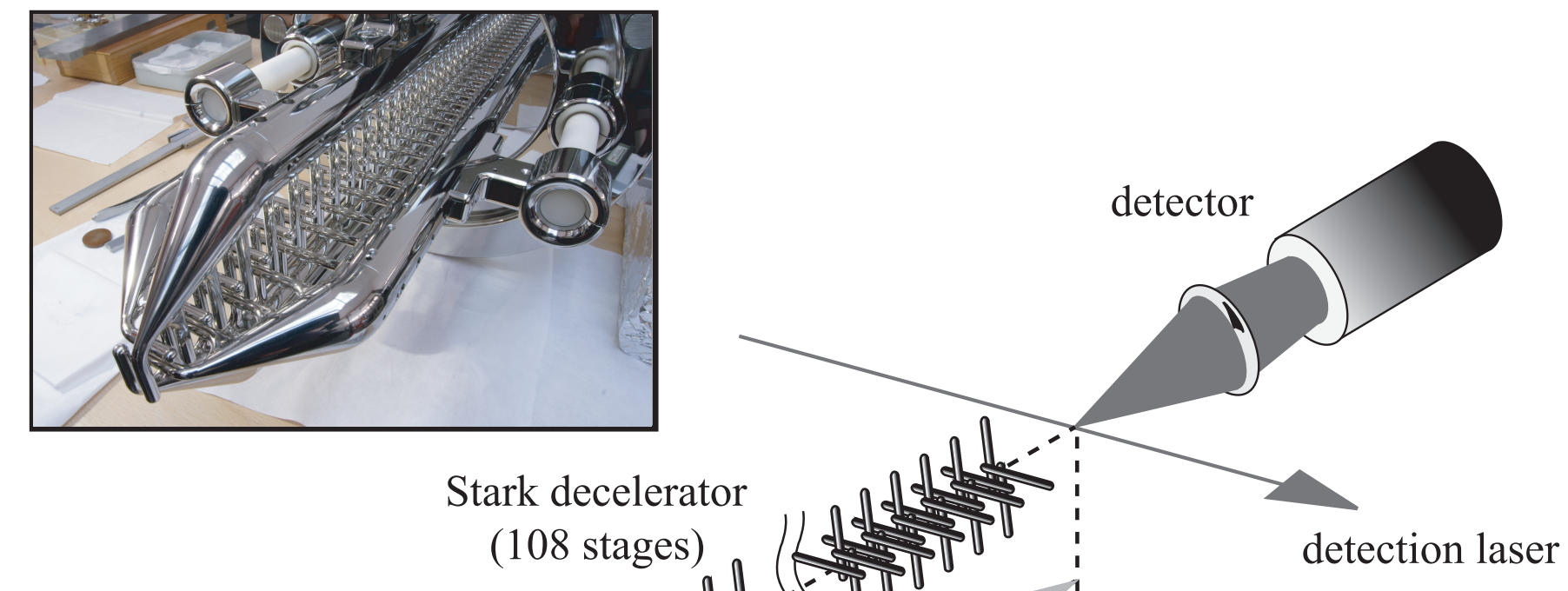

Stark decelerator

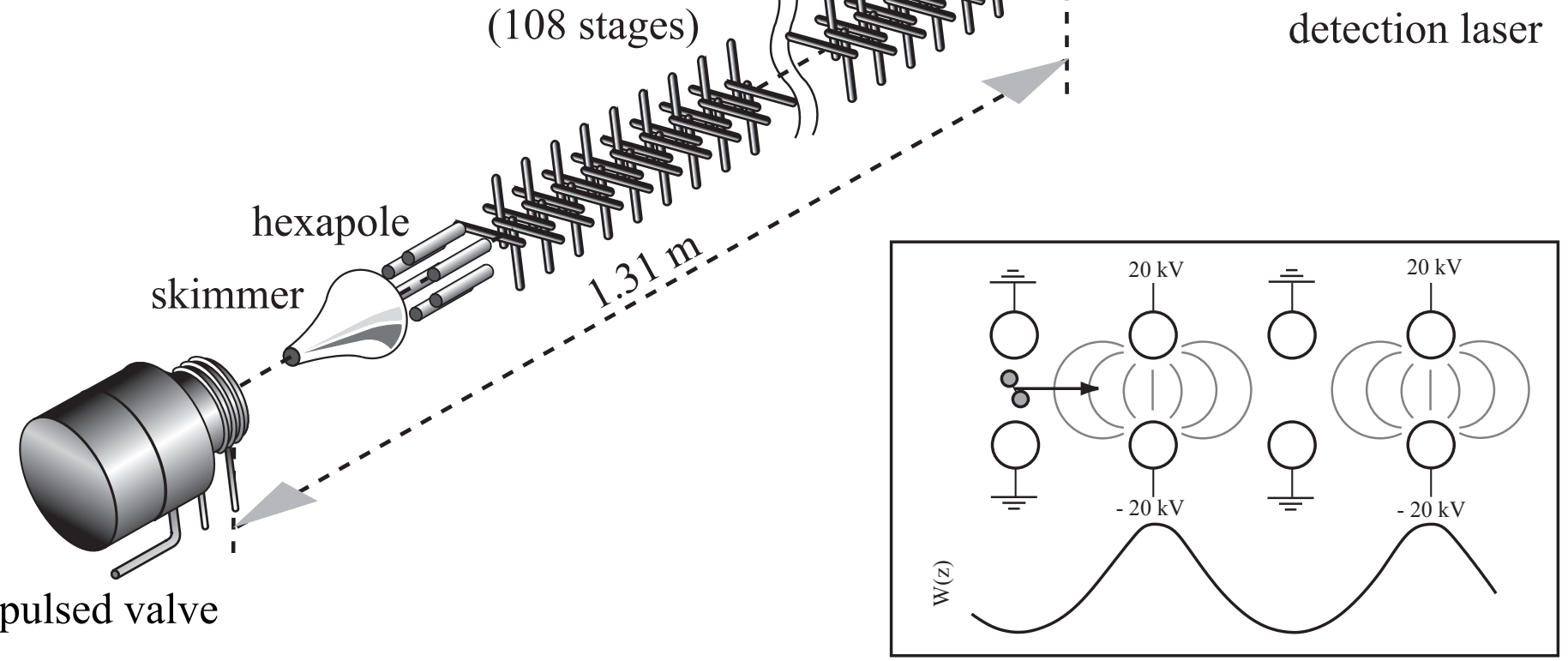



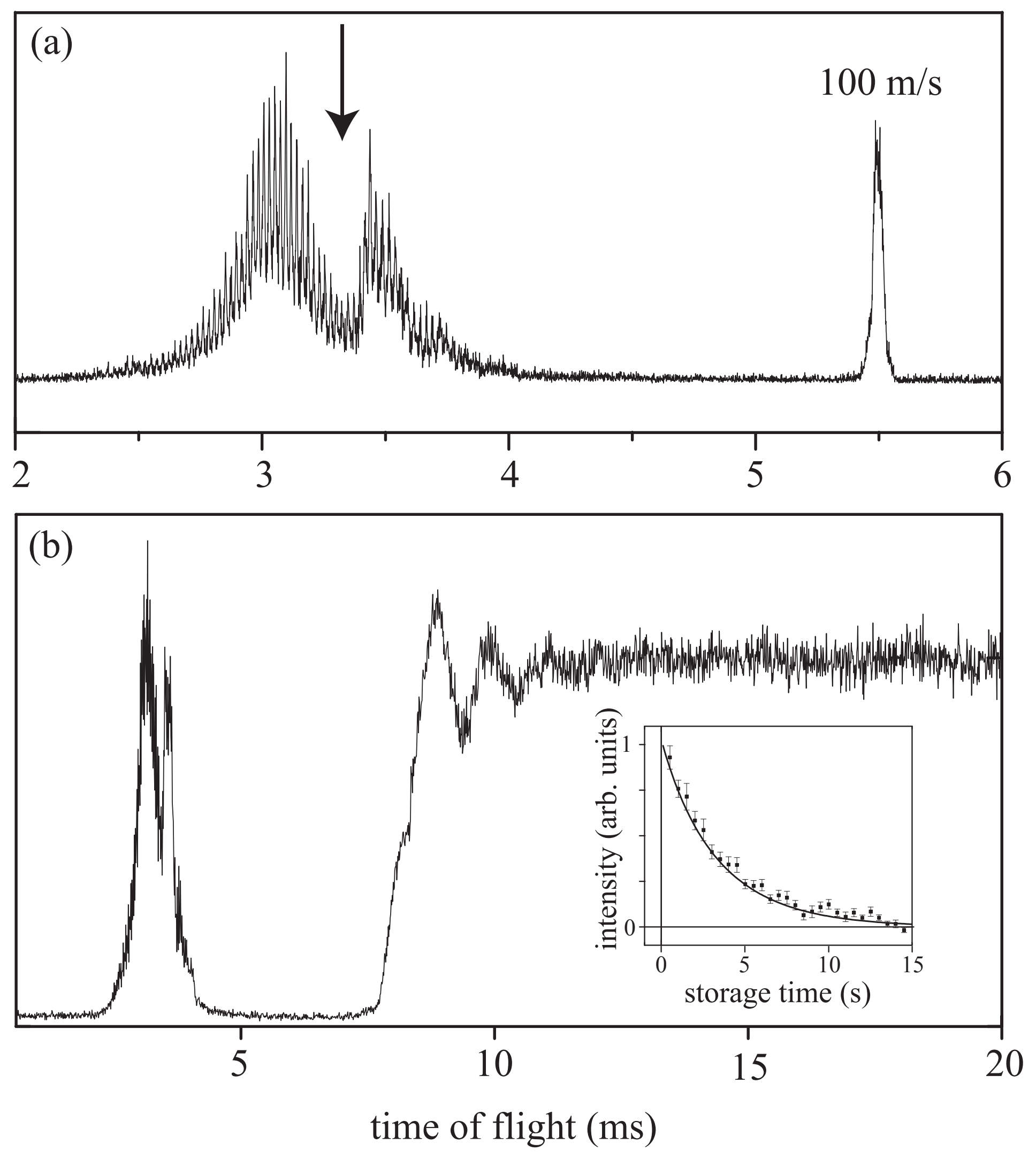
(a)

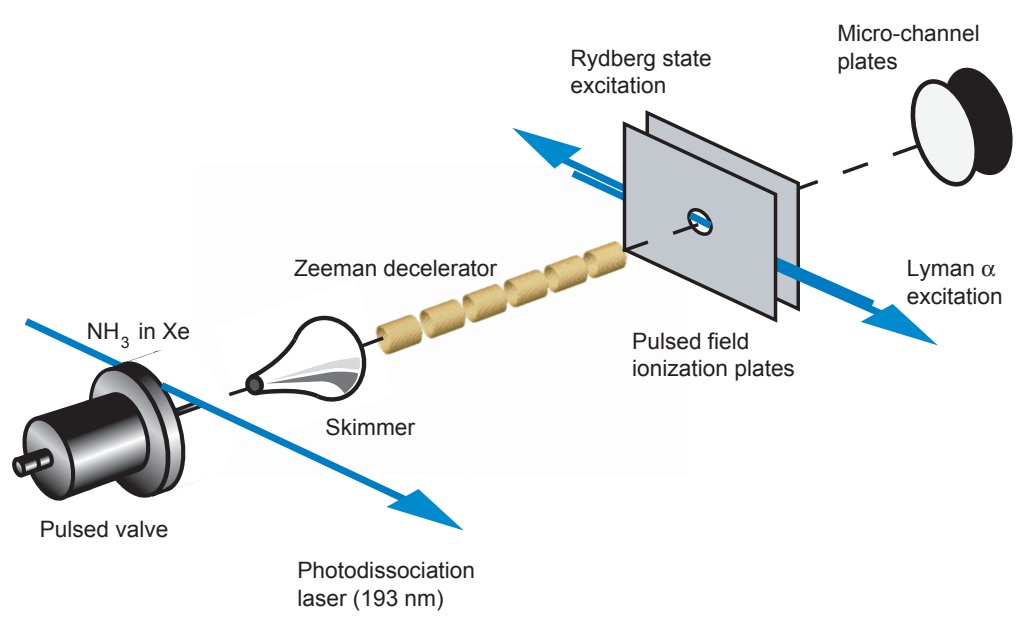

(b)

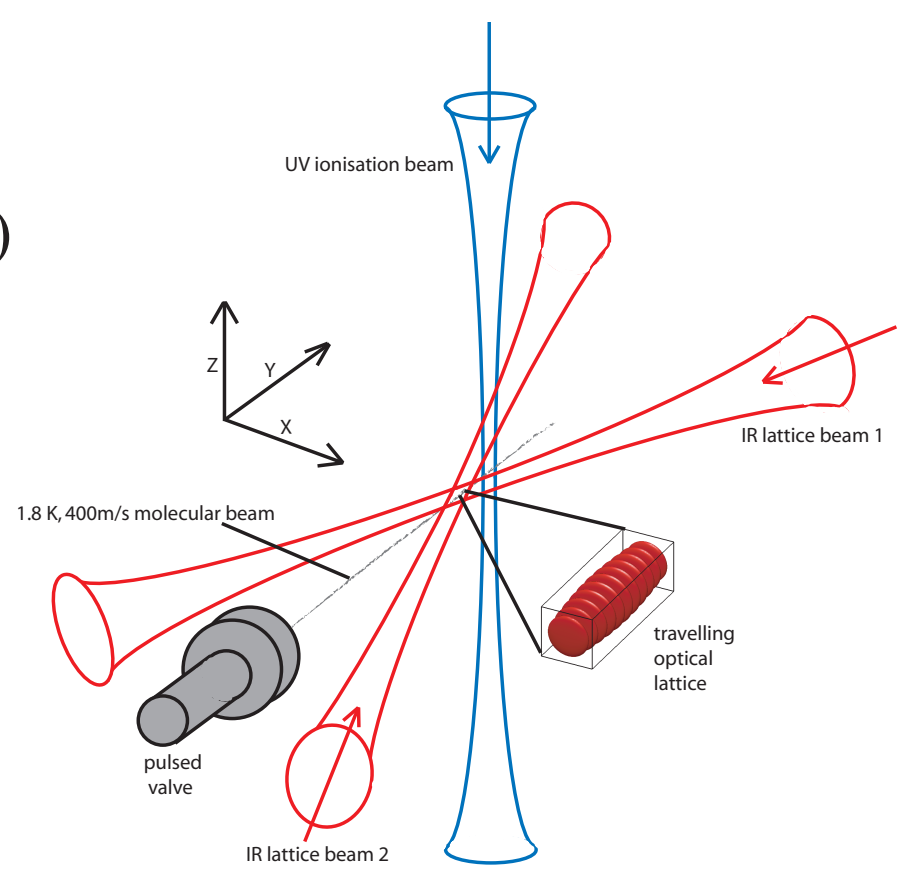




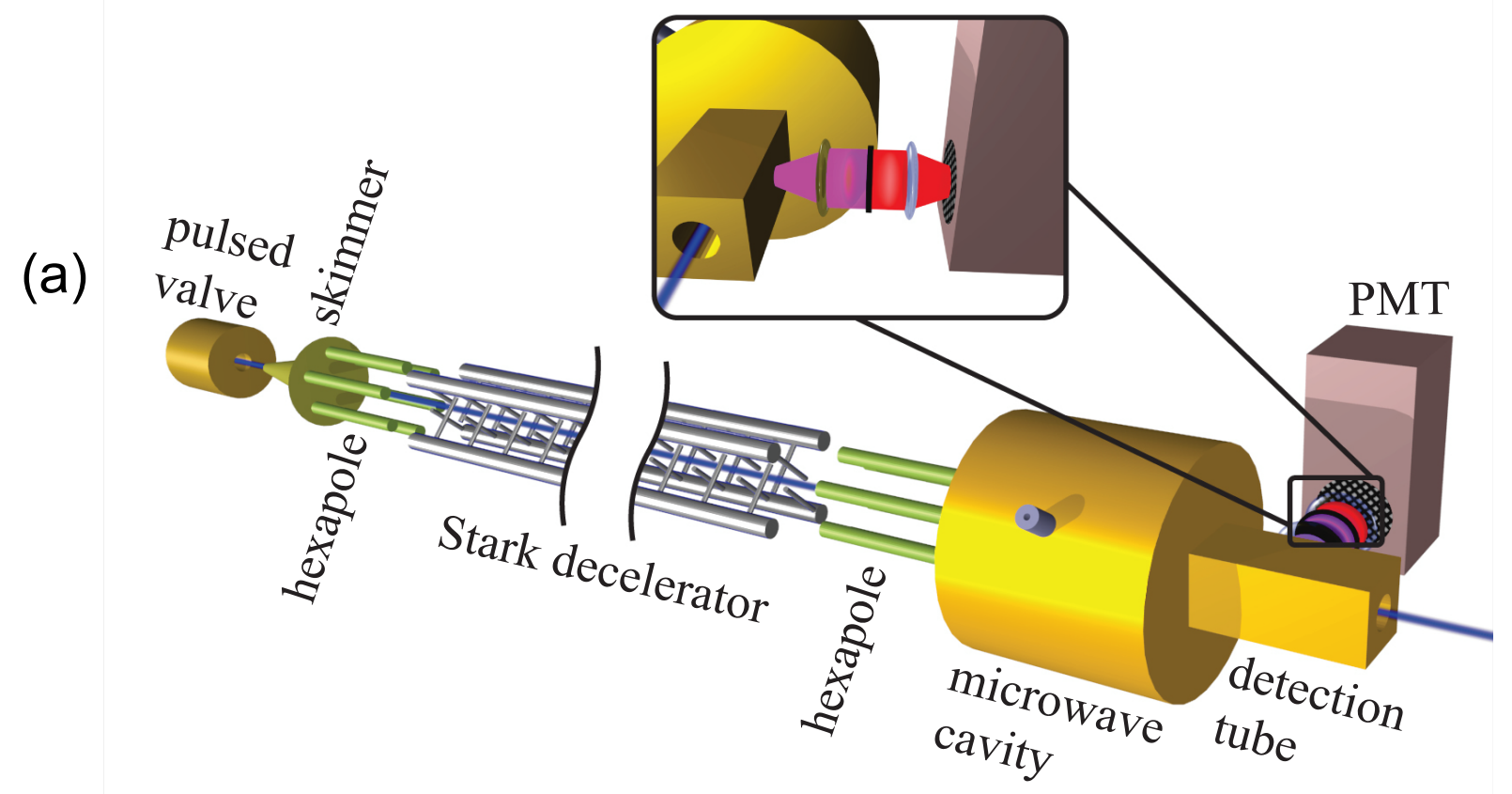

(b)

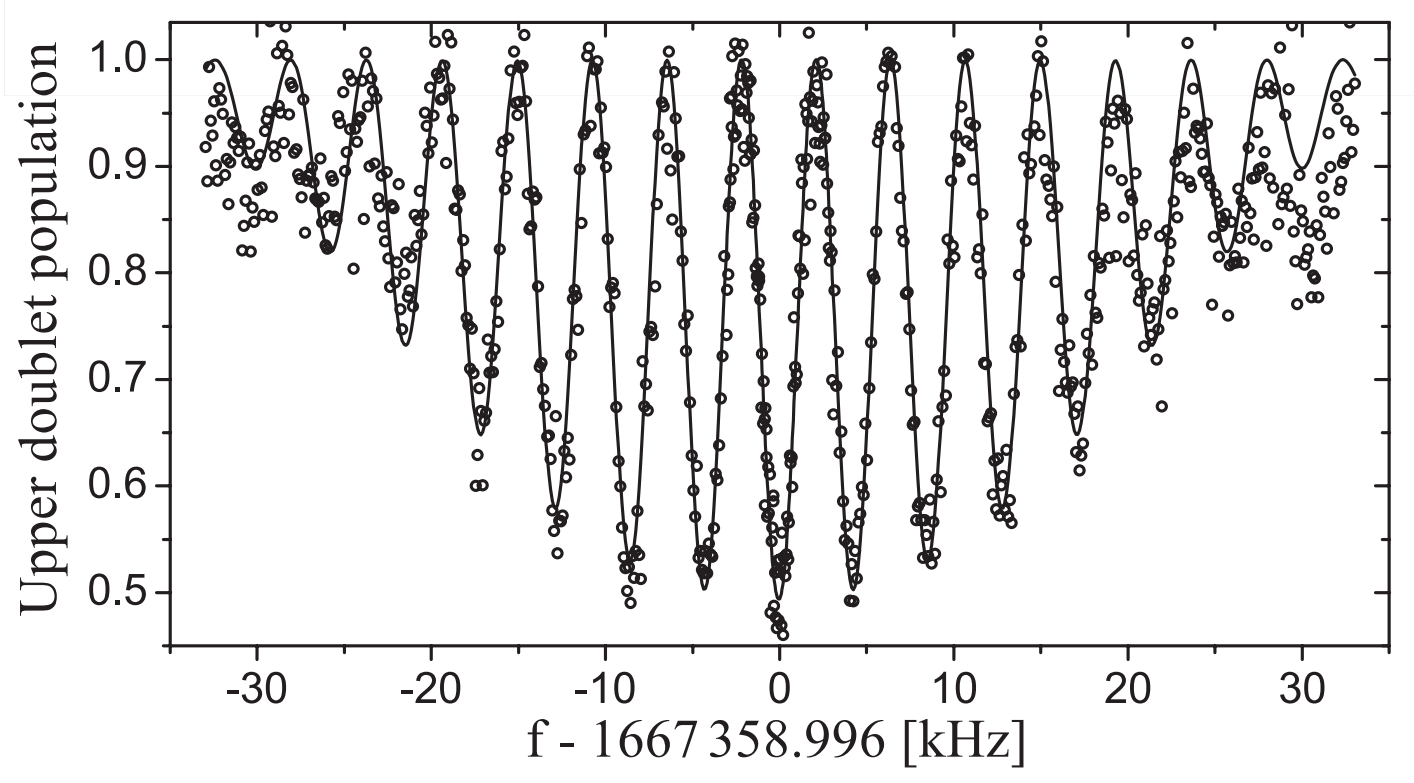




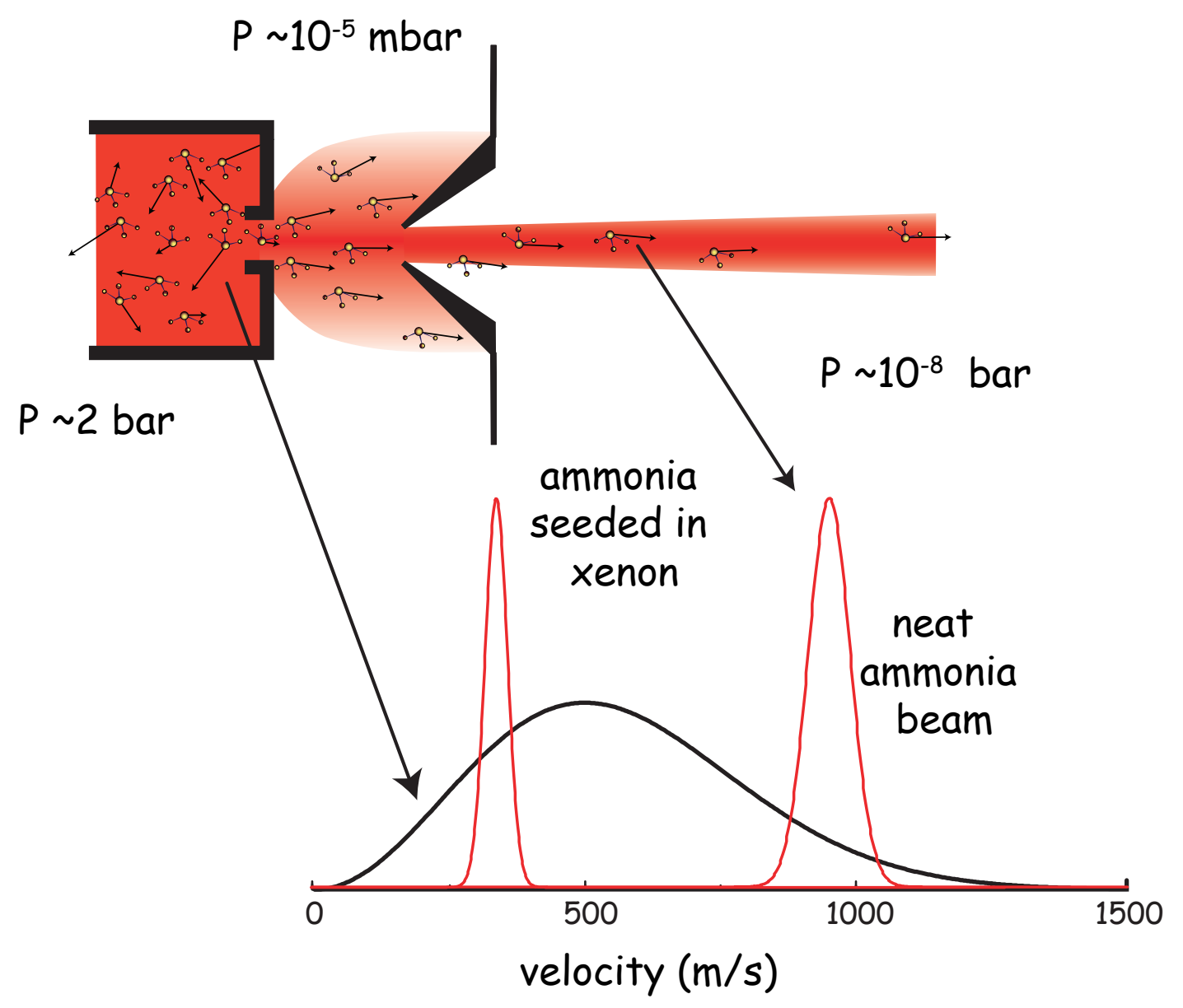




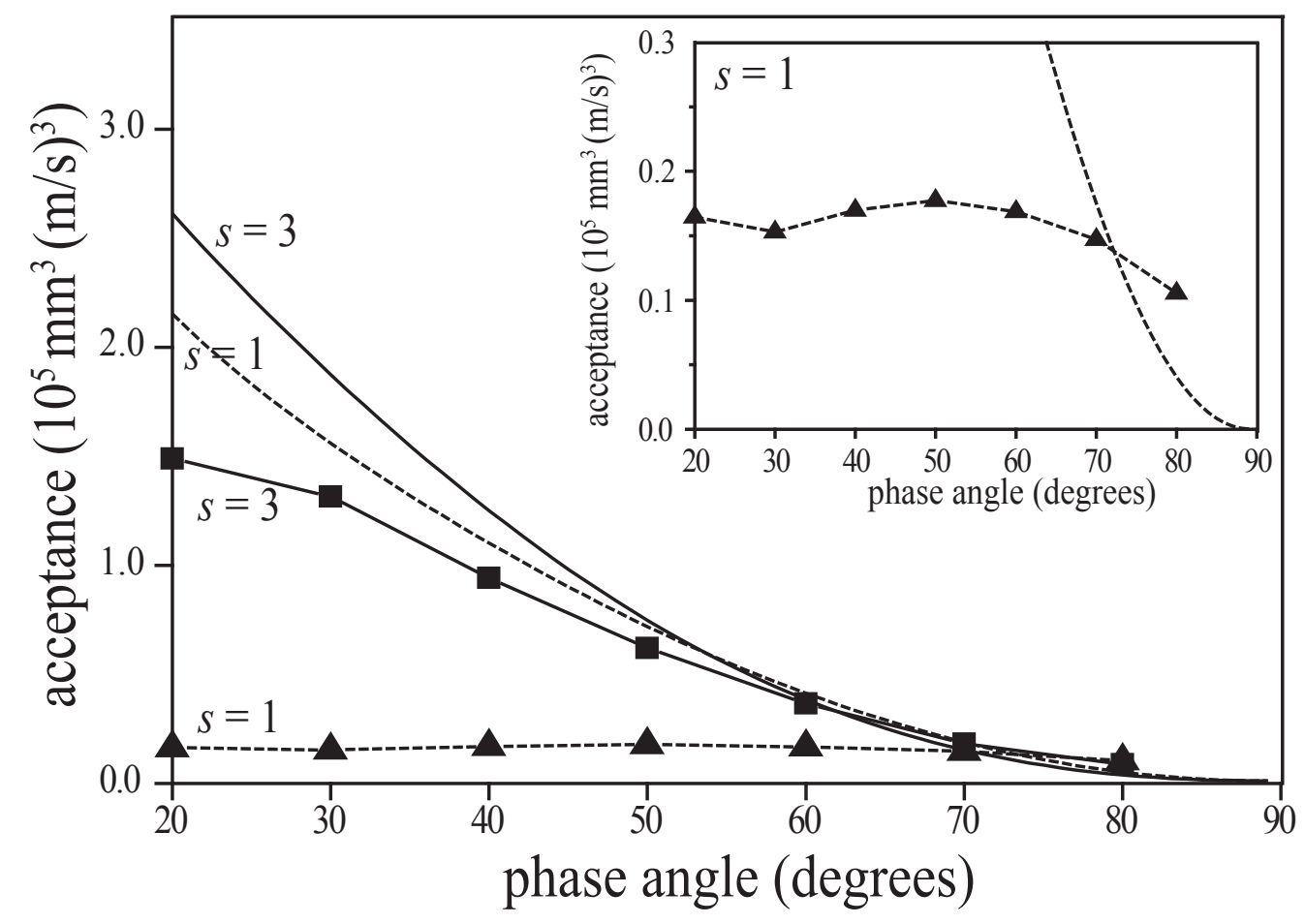


(a)

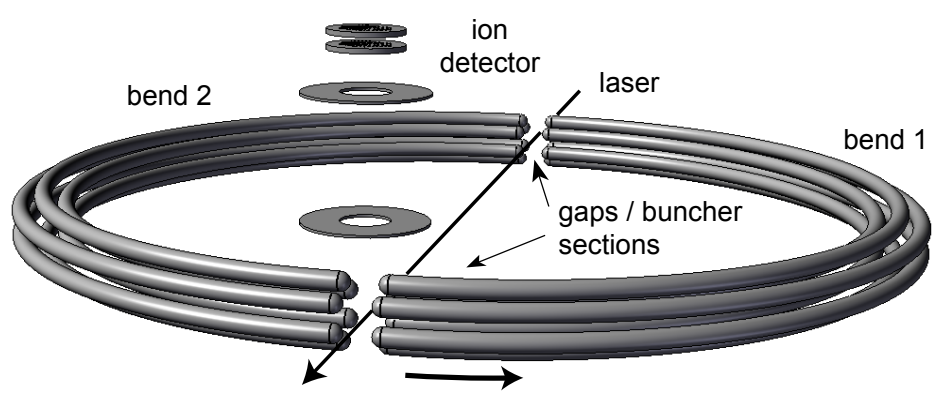

(b)

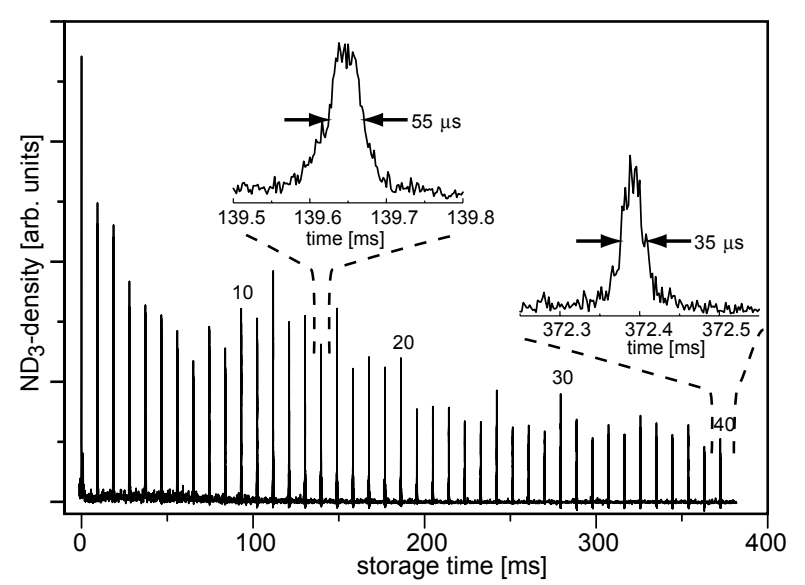

